\title{
Controlled islanding strategy for power systems based on flexible semi-supervised spectral clustering
}

\begin{abstract}
Islanding strategy is a self-healing method applying to power systems as the final step to prevent wide area blackouts. To suggest an effective islanding method, several parameters should be considered. Without those considerations, there is high possibility that generated islands will not be stable enough and will collapse soon. In this paper, a new method based on flexible semi-supervised spectral clustering is proposed to handle multiple constraints in order to guarantee the stability of generated islands. This method is also capable of handling and tackling various satisfaction degree of each constraint. Hence, the best solutions are achieved with respect to each defined constraint and its level of importance. The efficiency of this method for power systems is shown by applying it on the IEEE 39-buses network.
\end{abstract}

Keyword: Controlled islanding; Flexible spectral clustering; Blackout prevention 